\title{
PW03-034 - How to classify autoinflammatory diseases?
}

\author{
G Grateau ${ }^{1^{*}}$, V Hentgen², K Stankovic ${ }^{3}$ I Jéru ${ }^{4}$, S Amselem ${ }^{4}$, O Steichen ${ }^{1}$ \\ From 7th Congress of International Society of Systemic Auto-Inflammatory Diseases (ISSAID) \\ Lausanne, Switerland. 22-26 May 2013
}

\section{Introduction}

Definitions and classifications of autoinflammatory diseases have been multiple. Their succession highlights the advances in our understanding of the innate immune system, especially the role of interleukin $1 \beta$ and the inflammasome. However, these definitions and classifications face a number of structure and content issues.

\section{Objectives}

To propose a novel definition of autoinflammatory diseases and to challenge the global classification of inflammatory diseases.

\section{Methods}

We appeal to the desirable characteristics of classification systems (exhaustiveness, disjointness, naturalness, usefulness) and to a critical analysis of the notion of continuum.

\section{Results}

We propose a clinically-oriented definition: "autoinflammatory diseases are diseases with clinical signs of inflammation, associated with elevated acute phase reactants and due to a dysfunction in the innate immune system, genetically determined or triggered by an endogenous factor".

It is hard to find natural properties able to underlie a useful classification of autoinflammatory diseases, and inflammatory diseases as a whole, into disjoint and exhaustive categories. The notion of continuum is therefore appealing. However, a single continuum from purely autoinflammatory to purely autoimmune diseases oversimplifies, and even distorts, reality. How to locate, for instance, the disease caused by a deletion in PLCG2 (the gene encoding phospholipase $\mathrm{C} \gamma 2$ ) that associates autoinflammatory symptoms to both common variable immunodeficiency and autoimmune features? Here we have an overactivation of both the innate and the adaptive immune system, associated with a deficiency of the adaptive immune system.

More than one dimension is needed to properly represent the immunological dysfunctions underlying inflammatory diseases. Furthermore, a classification of inflammatory diseases should also make sense of the clinical, pathological and biological phenotypes.

\section{Conclusion}

To be adequate and useful, a definition of autoinflammatory diseases and a classification of inflammatory diseases must take the multiple facets of reality into account, including clinical features. This can be done within a continuum only if it is multidimensional.

\section{Disclosure of interest \\ None declared.}

\section{Authors' details}

${ }^{1}$ Université Pierre Et Marie Curie Paris 6, Paris, France. ${ }^{2}$ Pédiatrie, Centre Hospitalier, Le Chesnay, France. ${ }^{3}$ Hôpital Tenon, France. ${ }^{4}$ Génétique, INSERM UMR 933, Paris, France.

Published: 8 November 2013

doi:10.1186/1546-0096-11-S1-A260

Cite this article as: Grateau et al:: PW03-034 - How to classify autoinflammatory diseases? Pediatric Rheumatology 2013 11(Suppl 1):A260.

${ }^{1}$ Université Pierre Et Marie Curie Paris 6, Paris, France

Full list of author information is available at the end of the article 